\title{
Review
}

\section{Dissecting the role of ubiquitylation in the DNA damage response checkpoint in $\mathrm{G} 2$}

\author{
F Bassermann ${ }^{\star, 1}$ and M Pagano ${ }^{\star, 2,3}$
}

Maintenance of genomic integrity is one of the fundamental biological properties shared by all living organisms. To counterbalance deleterious and potentially mutagenic effects of omnipresent DNA damaging assaults, organisms have developed a network of genome surveillance and maintenance pathways known as the DNA damage response. In eukaryotes, the orchestration of cell-cycle checkpoints, DNA damage repair, and apoptosis in response to DNA damage relies on posttranslational modifications of key regulatory proteins. Although the role of phosphorylation in these pathways is relatively well established, the significance of ubiquitylation has only recently emerged. In this review, we survey current research on the ubiquitin-proteasome system, focusing on the DNA damage response in the G2 phase of the cell cycle and two prominent classes of ubiquitin ligases, the SCF- and APC/C complexes. These ubiquitin ligases are reviewed with regard to their function in activating, maintaining, and terminating the checkpoint and in light of increasing evidence that suggests a dynamic balance of substrate ubiquitylation and deubiquitylation. We further discuss the impact of defective G2 checkpoint signaling on genomic stability and cancer risk, highlighting strategies for targeted antitumor drug discovery.

Cell Death and Differentiation (2010) 17, 78-85; doi:10.1038/cdd.2009.104; published online 14 August 2009

Proper duplication of chromosomes is critical to cell function and the survival of organisms. DNA damage caused by endogenous (e.g. replication stress or free radicals) or exogenous factors (e.g. exposure to UV light or ionizing radiation) is a constant threat to the faithful transfer of genomic information. To prevent the mutagenic effects of DNA damage, eukaryotic cells respond to damage by activating a checkpoint response that arrests cell-cycle progression to allow for damage repair before the next stage of cell division. Alternatively, if the damage is too excessive, senescence or apoptosis can be induced. ${ }^{1-3}$

Although the G1/S and the intra-S phase checkpoints prevent cells from unfaithful replication of their chromosomes, the G2 checkpoint allows repair of DNA damage just before mitosis. Checkpoint induction and DNA repair are thus closely interconnected to control genomic stability and prevent cells from undergoing malignant transformation., ${ }^{4,5}$ There are several DNA repair pathways in human cells, including mismatch repair, base excision repair, nucleotide excision repair, homologous recombination (HR), non-homologous end joining, and translesion synthesis. These pathways are triggered by different types of DNA damage and are differentially active depending on the cell type and cell-cycle phase. This review focuses on the cellular response to double strand breaks (DSBs) in the G2 phase of the cell cycle. DSBs are typically repaired by either non-homologous end joining (which is error prone) or HR, depending on the availability of sister chromatids. Therefore, the mechanism of choice in G2 is HR. Briefly, the central reaction of $H R$ is a search for homology and DNA strand invasion by Rad51-ssDNA (single stranded DNA) presynaptic filaments, which initiate HR and form DNA structures called D-loops. These events are downstream to ATM activation, which occurs through the MRE11-Rad50-NBS1 (MRN) complex and DSB resection, which exposes $3^{\prime}$ overhangs of ssDNA. Eventually, DNA synthesis (using the undamaged chromatid as a template) is performed to span the break site, thus generating two intact, separate chromatids [reviewed in References ${ }^{4,6,7}$ ].

During the initiation of the G2 checkpoint, numerous proteins, including the phosphoinositide 3-kinase-related kinase ATM, ATR, and DNA-PK, collaborate to activate the DNA damage response signaling network. ${ }^{8-10}$ In terms of checkpoint activation, these interwoven signaling cascades ultimately focus on the master regulator of mitotic entry, Cdk1, whose activity must be inhibited to arrest the cell cycle. ${ }^{11}$ Although many of the proteins involved in the G2 cell-cycle block are known, our knowledge of the crosstalk between these players still remains fragmentary. Timely response to genotoxic insults requires mechanisms producing an immediate impact, making this process largely reliant on different

\footnotetext{
${ }^{1}$ Department of Medicine III, Technical University of Munich, Munich, Germany; ${ }^{2}$ Department of Pathology, NYU Cancer Institute, Smilow Research Center, New York University School of Medicine, New York, NY, USA and ${ }^{3}$ Howard Hughes Medical Institute

${ }^{*}$ Corresponding authors: F Bassermann, Department of Medicine III (Hematology/Oncology), Technical University of Munich, Ismaninger Strasse 22, 81675 Munich, Germany. Tel: + 49894140 5038; Fax: + 49894140 4879; E-mail: florian.bassermann@ Irz.tum.de or M Pagano, Department of Pathology, New York University School of Medicine, 522 First Ave., SRB 1107, New York, NY 10016, USA. Tel: +1 212263 5332; Fax: +1 212263 5107; E-mail: michele.pagano@ nyumc.org Keywords: ubiquitylation; DNA damage response; checkpoint; G2 phase; cell cycle

Abbreviations: DSBs, double strand breaks; UPS, The ubiquitin-proteasome system; HR, homologous recombination; ssDNA, single stranded DNA; SCF, Skp1/Cul1/ F-box protein

Received 31.3.09; revised 03.6.09; accepted 30.6.09; Edited by G Melino; published online 14.8.09
} 
posttranslational modifications. Next to protein phosphorylation/dephosphorylation, for which a role in the G2 DNA damage response is relatively well established, protein ubiquitylation/deubiquitylation has only recently become more appreciated in the DNA damage response. It is becoming evident that both mechanisms closely cooperate, thus establishing the highly organized network needed for a timely and accurate response. Recent advances in this field, with a focus on the SCF and APC/C ubiquitin ligases, are discussed herein.

\section{SCF and APC/C ubiquitin ligases}

The ubiquitin-proteasome system (UPS) drives the cell cycle in a unidirectional and irreversible manner by targeting key cell-cycle regulators for timely degradation.

Two ubiquitylation complexes are intimately involved in this process, namely the SCF (Skp1/Cul1/F-box protein) complexes and the APC/C (anaphase-promoting complex/cyclosome). SCF and APC/C complexes are evolutionarily related, multiprotein complexes, with both containing a protein with cullin homology (Cul1 in the SCF complex, Apc2 in APC/C) and a RING finger protein (Roc1/Rbx1/Hrt1 in the SCF complex and Apc11 in APC/C) ${ }^{12-14}$ (Figure 1). The complexes differ in terms of their activity profile throughout the unperturbed cell cycle, with SCF complexes being active throughout the cell cycle and the APC/C being active only from mitosis to late $\mathrm{G} 1$.

F-box proteins (69 in humans) are the variable components of SCF complexes, which recruit substrates to the SCF core, thus providing specificity. They are divided into three groups,
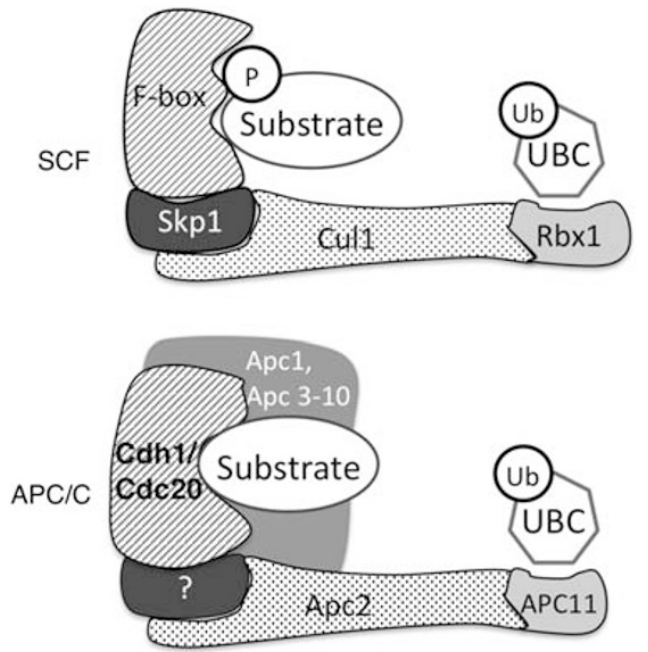

Figure 1 Structure of SCF and APC/C ubiquitin ligases. Both ubiquitin ligases share a Cullin (dotted) subunit and a RING finger (light gray) protein, which mediates binding to the ubiquitin conjugating enzyme (UBC). The substrate-binding subunits are the variable components in each family of ligases (dashed) and mediate substrate-binding specificity. Cdh1 and Cdc20 are the variable components of the APC/C, whereas SCF complexes are characterized by a large class of substrate-binding components, the F-box proteins (69 in humans). SCF ligases typically recognize phosphorylated substrates, whereas the APC/C targets specific motifs present in the primary sequence of the substrate. In addition to Cdh1 and Cdc20, some substrates are also recognized though the contribution of other APC/C subunits (e.g. Apc10) based on the presence of WD-40 domains (FBXWs), leucinerich repeats (FBXLs), or other non-WD-40/non-leucine-rich repeats protein-interaction domains (FBXOs). Notably, only 9 of the 69 SCF complexes have been matched to relevant substrates. ${ }^{12,15}$ In addition, SCF-like ubiquitin ligases have been described. For instance, the F-box-like protein, nuclear interaction partner of ALK, forms an SCF-like complex that has been implicated in the regulation of the $\mathrm{G} 2 / \mathrm{M}$ transition. ${ }^{16,17}$

The APC/C complex comes in two forms, defined by either of two activating subunits/substrate-binding determinants: Cdc20 and Cdh1 (Figure 1). Although Cdc20 is necessary for progression through mitosis (i.e. promoting degradation of Securin to allow Separase function) and mitotic exit (i.e. promoting cyclin degradation to inactivate Cdk1), Cdh1 maintains the G0/G1 state by keeping CDK activities low (i.e. promoting the degradation of cyclins, Cdc25A, Plk1, Skp2, Cks1, etc.) and targeting key components of the DNA replication machinery (e.g. Geminin, Cdc6, Tk1, and Tmpk). A key difference between SCF and APC/C lies in their mode of substrate recognition. SCF complexes, in the majority of defined SCF systems, recognize substrates only when phosphorylated, whereas APC/C mostly recognizes unmodified, specific motifs present in the primary sequence of the substrate (e.g. D-box, KEN-box, A-box, etc.). In addition, unlike SCF, APC/C is regulated by CDK-mediated phosphorylation, with $A P C / C^{C d h 1}$ being inactivated in $S, G 2$, and early $\mathrm{M}$, and $\mathrm{APC} / \mathrm{C}^{\mathrm{Cdc20}}$ being activated in mitosis. ${ }^{13} \mathrm{SCF}$ and APC/C complexes also communicate and establish feedback among each other. For instance, during $\mathrm{G} 1, \mathrm{APC} / \mathrm{C}^{\mathrm{Cdh} 1}$ promotes the degradation of Skp2 and its cofactor Cks1, and SCF contributes to the degradation of Cdh1 in early $S$ phase. ${ }^{18-20}$ Thus, a complex network of positive and negative feedback loops is established between kinases and ligases, providing the high level of specificity and flexibility of the system.

\section{Establishment and maintenance of the G2 checkpoint}

At the heart of the G2 DNA damage checkpoint lies the Cdk1 kinase (also known as Cdc2), the only CDK in mammals that is essential for viability. On DNA damage, Cdk1 must be inhibited to arrest the cell cycle and inhibit mitotic entry. Cdk1 activity is regulated by inhibitory phosphorylation (on Thr 14 and Tyr 15 in human), which is tightly controlled by the Wee1 and Mik1/Myt1 kinases and the Cdc25 family of phosaphatases. In addition, the CDK inhibitory proteins p21 and p27 negatively control Cdk1 activity. ${ }^{21}$ After the formation of DSBs, ATR and ATM activate Chk1 and Chk2, respectively. In turn, these two kinases induce the degradation of Cdc25A and promote the stabilization of p53, leading to the induction of p21 (reviewed in References ${ }^{2,3,11}$ ). Notably, ATR requires the checkpoint mediator molecule Claspin to activate Chk1. ${ }^{22}$ Two major ubiquitin ligases are integral to this process: $\mathrm{SCF}^{\beta \mathrm{TrCP}}$ and $\mathrm{APC} / \mathrm{C}^{\mathrm{Cdh} 1}$.

$\mathrm{SCF}^{\beta \mathrm{TrCP}}$. One essential G2 checkpoint hub is operated by the $\mathrm{SCF}^{\beta}{ }^{\beta \mathrm{TrCP}}$ ligase. Mammals express two distinct paralogs of $\beta \operatorname{TrCP}$, which are biochemically indistinguishable: $\beta \operatorname{TrCP} 1$ (also known as FBXW1, FBW1A, and FWD1) and $\beta$ TrCP2 
(also known as FBXW11, FBW11, FBXW1B, FBX1B, and HOS). ${ }^{15}$ We will therefore use the term $\beta$ TrCP to refer to both, unless specified. The key function of $\mathrm{SCF}^{\beta \mathrm{TrCP}}$ in $\mathrm{G} 2$ stems from its ability to regulate the checkpoint both positively and negatively. This dual function is intimately linked to the mode of substrate recognition by the $\mathrm{SCF}^{\beta \operatorname{TrCP}}$ ligase, which is governed by phosphorylation. $\beta \mathrm{TrCP}$ recognizes a canonical DSGXXS degradation motif (degron) or related variants (for example, the DDG, EEG, SSG, or TSGXXS/E/D motifs) in which the serine or threonine residues are phosphorylated by specific kinases to allow binding to $\beta \operatorname{TrCP}$. The usage of substrate phosphorylation imparts not only exquisite specificity but also functions as a means to regulate $\mathrm{SCF}^{\beta \mathrm{TrCP}}$, which is present and active throughout the cell cycle. Three kinases are relevant for this process in G2: Plk1, Chk1, and Chk2. Plk1, a prominent pro-mitotic kinase, initiates checkpoint recovery by directly phosphorylating the checkpoint molecules Claspin and Wee1, thus marking them for degradation by $\mathrm{SCF}^{\beta \operatorname{TrCP}}{ }^{23}$ (Checkpoint recovery is discussed in more detail below.) Chk1- and Chk2-mediated phosphorylation activates the checkpoint by mediating $\mathrm{SCF}^{\beta \operatorname{TrCP}}$-dependent degradation of the Cdk1 activator Cdc25A. ${ }^{24,25}$

In mammals, there are three Cdc25 family members (Cdc25A, B, and C), which collaborate in coordinating the timely activation of multiple cyclin-CDK complexes at different phases of the cell cycle. ${ }^{26-29}$ Cdc25B and $C$ are regulators of the $\mathrm{G} 2 / \mathrm{M}$ transition by virtue of their ability to activate cyclin A, B-Cdk1. ${ }^{30,31}$ In contrast, Cdc25A controls not only the $\mathrm{G} 2 / \mathrm{M}$ transition by regulating $\mathrm{Cdk} 1,{ }^{24,32}$ but it also controls the $\mathrm{G} 1 / \mathrm{S}$ transition by participating in the regulation of Cyclin E/A-Cdk2 activity. ${ }^{33}$ Notably, Cdc25A is the only Cdc25 family member essential for the mammalian cell cycle. ${ }^{34,35}$ The mode of phosphorylation of Cdc25A has been subject to extensive scrutiny, and a complex picture, in which multiple phosphorylation events can both promote and protect Cdc25A from ubiquitin-mediated proteolysis, has recently emerged (for a review see Reference ${ }^{36}$ ). With regard to the $\mathrm{G} 2$ checkpoint response, the data support a model in which a Chk1/2mediated priming phosphorylation is a prerequisite for the subsequent phosphorylation of Cdc25A (by a still unidentified kinase(s)), which creates the phosphodegron for $\mathrm{SCF}^{\beta \operatorname{TrCP}}$ (Figure 2). Cdc25A protein is overexpressed in a variety of human cancers, including head and neck, liver, thyroid breast, ovary, and lung cancers, and such overexpression often correlates with poor prognosis. ${ }^{28} \mathrm{~A}$ recent study using MMTV-Cdc25A transgenic mice showed that Cdc25A overexpression in the mouse mammary gland is insufficient to cause spontaneous tumors. ${ }^{37}$ However, the MMTV-Cdc25A transgene significantly accelerates MMTV-Ras- or MMTV$\mathrm{Neu}$-induced mammary tumorigenesis, with tumors showing mis-coordinated cell-cycle progression and enhanced genomic instability. Whether mutations within the degron of Cdc25A are the root of the high expression levels observed in certain tumors is presently unclear. Future studies applying knock-in models for the mutant forms of Cdc25A and sequence analyses in primary tumor samples will help to further unravel the role of Cdc25A degradation in tumorigenesis and indicate whether Cdc25A is a promising target gene for molecular therapy.

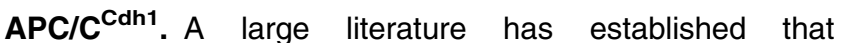
$\mathrm{APC} / \mathrm{C}^{\mathrm{Cdh} 1}$ primarily functions during late mitosis and in G1 of the unperturbed cell cycle to ensure that certain positive regulators of the cell cycle do not accumulate prematurely. ${ }^{13,14}$ At the G1/S transition, cyclin A-Cdk1- and cyclin A-Cdk2-mediated phosphorylation of Cdh1 results in dissociation of Cdh1 from the APC/C core and, thus, inactivation of $\mathrm{APC} / \mathrm{C}^{\mathrm{Cdh} 1}{ }^{38-40}$ The consequent stabilization of substrates promotes progression into $S$ phase. Binding to Emi1 (an APC/C inhibitor) and degradation of both Cdh1 and Ubc10 (a ubiquitin conjugating enzyme, which cooperates with $\mathrm{APC} / \mathrm{C}$ ) represent further mechanisms to inhibit $\mathrm{APC} /$ $\mathrm{C}^{\mathrm{Cdh} 1}$ at $\mathrm{G} 1 / \mathrm{S}^{20,41-43}$ On progression into $\mathrm{G} 2$, both $\mathrm{Cdh} 1$ and Ubc10 re-accumulate, whereas $A P C / C^{C d h 1}$ remains in an inactive state as a consequence of CDK-dependent phosphorylation of Cdh1 and the presence of Emi1.

The first hints of a role for $A P C / C^{C d h} 1$ in the G2 DNA damage checkpoint came from observations in chicken DT40 cells, in which re-association of Cdh1 with Cdc27 (one of the core components of the APC/C) was observed in response to genotoxic stresses. This re-association was accompanied by an increase in APC/C ubiquitylation activity. ${ }^{44}$ Subsequently, reactivation of $A P C / C^{C d h 1}$ in response to DNA damage was also observed during $\mathrm{G} 2$ in mammalian cells and shown to be required for the degradation of $\mathrm{Plk}_{1}{ }^{45}$ (Figure 2). Plk1 is required for mitotic entry, and its kinase activity triggers the degradation of the Cdk1 inhibitors Wee1 and Claspin through SCF $^{\beta T r C P}{ }^{46-49}$ Indeed, cells expressing a mutant form of Plk1 that cannot be recognized by APC/C ${ }^{\mathrm{Cdh} 1}$ (and thus cannot be degraded) showed a greater tendency to escape from the DNA damage checkpoint and enter mitosis despite the presence of DNA damage foci. ${ }^{45}$ Intriguingly, although Cdh1 is not an essential gene, recent findings from a conditional knockout approach showed increased genomic instability in mouse embryonic fibroblasts lacking $C d h 1 .^{50,51}$ This phenotype may be the consequence of an impaired DNA damage checkpoint.

The obvious question of how APC/C ${ }^{\mathrm{Cdh} 1}$ becomes reactivated in the DNA-damaged cells in G2, when Cdk activity is high, was solved by the identification of Cdc14B as the phosphatase that specifically dephosphorylates Cdh1 upon DNA damage in G2, thus removing a major restraint of Cdh1 activity. This event is preceded by the translocation of Cdc14B from the nucleolus to the nucleus. ${ }^{45}$ Here, an analogy to the regulation of mitotic exit in budding yeast is notable, because in early anaphase the release of Cdc14 from the nucleoli occurs through the sequential action of two regulatory networks, the Cdc fourteen early anaphase release network (FEAR) and the mitotic exit network (MEN). ${ }^{52-54}$ Significantly, some components of both pathways are conserved in mammals, but, with the exception of Plk1 (the ortholog of yeast Cdc5), the functions of these homologs are largely unknown.

A further question concerns the Cdh1 inhibitor Emi1. Does Emi1 inactivation contribute to the reactivation of $A P C / C^{C d h} 1$ in response to DNA damage? Initial evidence suggests that this is not the case (at least for the early response and initial maintenance of the cell-cycle block) and that, instead, two distinct pools of $\mathrm{APC} / \mathrm{C}^{\mathrm{Cdh} 1}$ exist in G2. One pool is inactive both in the presence and absence of genotoxic stress and is 


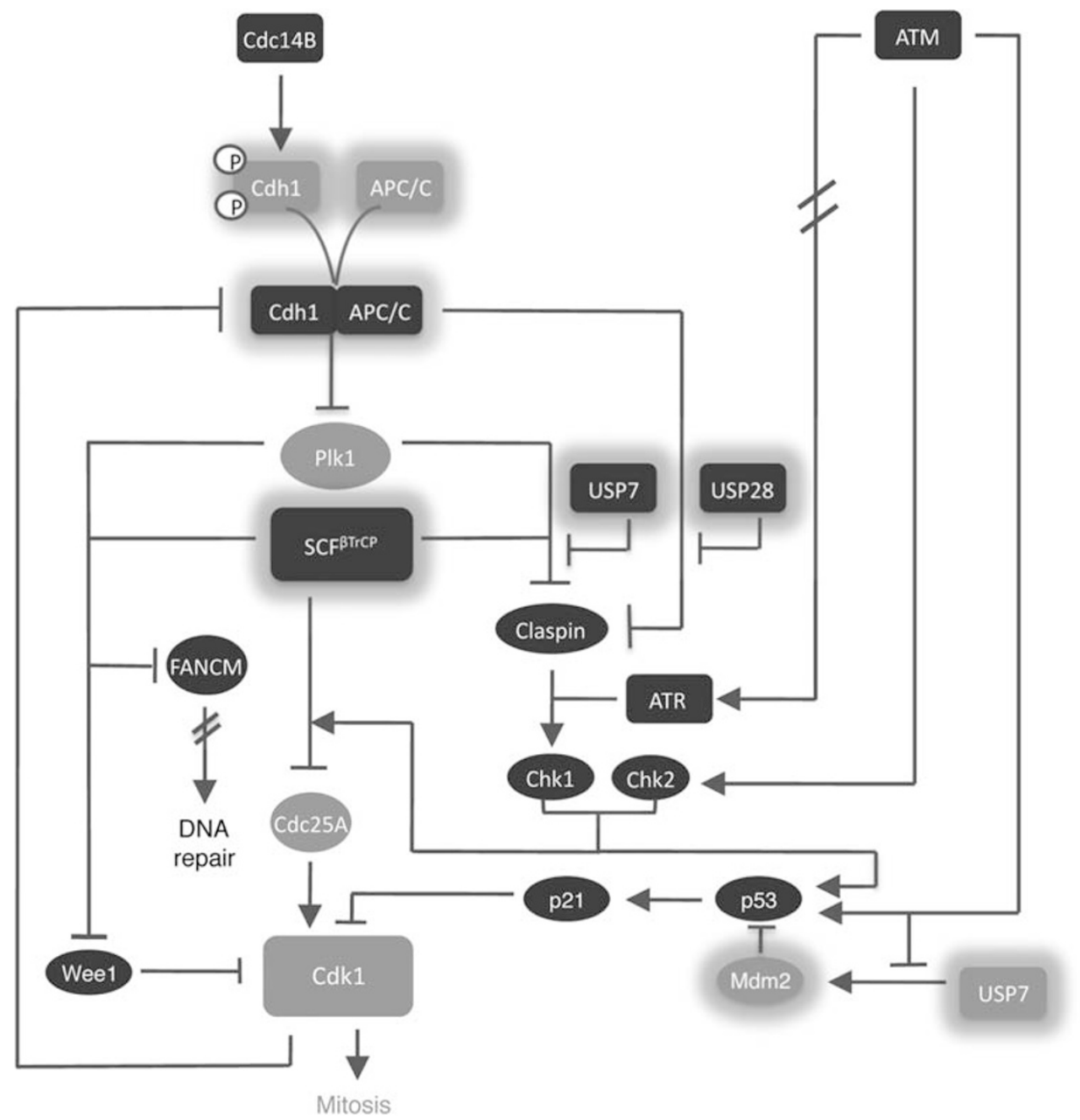

Figure 2 The role of ubiquitin ligases and deubiquitylating enzymes (DUBs) in controlling the G2 DNA damage response checkpoint. (Black indicates active forms, gray indicates inactive/degraded forms of the respected proteins or cellular events. Components of the UPS system are highlighted.) DNA damage during G2 triggers checkpoint activation signaling and eventually results in a cell-cycle block through inhibition of Cdk1. As part of the checkpoint initiation process, the phosphatase Cdc14B (after translocating to the nucleus from the nucleolus) activates $\mathrm{APC} / \mathrm{C}^{\mathrm{Cdh} 1}$ by dephosphorylating $\mathrm{Cdh} 1$, allowing binding to $\mathrm{APC} / \mathrm{C}$. APC/C ${ }^{\text {Cdh1 } 1}$ promotes the degradation of PIk1, a kinase required for mitotic entry by, among other functions, triggering the $\mathrm{SCF}^{\beta \mathrm{TrCP}}$-mediated degradation of Wee1 and Claspin, two negative regulators of Cdk1. Claspin is an adaptor protein required by the ATR kinase to activate Chk1, whose activity is a prerequisite for $\mathrm{SCF}^{\beta T r C P}$-mediated degradation of Cdc25A, an activator of Cdk1. During the G2 cell-cycle arrest, APC/C $C^{C d h 1}$ specifically induces the degradation of Plk1, but not other substrates, such as Claspin, because of the action of the deubiquitylating enzyme USP28. Additionally, USP7 counteracts Claspin ubiquitylation mediated by SCF ${ }^{\beta T r C P}$. In response to the DNA damage, ATM phosphorylates and activates Chk2, indirectly activates ATR, and phosphorylates Mdm2. The latter event lowers the affinity of Mdm2 for USP7, thus resulting in more rapid Mdm2 degradation. By this means, less p53 is ubiquitylated and degraded, resulting in the transcription of the Cdk1 inhibitor p21. Further stabilization of p53 is achieved by direct phosphorylation, which dissociates p53 from Mdm2, an event mediated by Chk1/2 and ATM

inhibited by Emi1, whereas a second pool is inhibited by phosphorylation and reactivated in response to DNA damage. In support of this hypothesis, certain substrates of APC/C ${ }^{\mathrm{Cdh} 1}$, which are known to be stabilized by Emi1 (e.g. cyclin A, cyclin $B$, and geminin), are not degraded despite $A P C / C^{C d h} 1$ re-activation upon DNA damage, and their stability does not change when either Cdh1 and/or USP28 (see below) is downregulated. ${ }^{45}$ In addition, Emi1 binding to APC/C is not influenced by genotoxic stresses. ${ }^{45} \mathrm{~A}$ recent report showed a p21-dependent downregulation of Emi1 in the late maintenance stage of the G2 checkpoint and linked this mechanism to the activation of $\mathrm{APC} / \mathrm{C}^{\mathrm{Cdh} 1} .^{55}$ Emi1 inactivation may thus contribute to G2 checkpoint maintenance, particularly after extensive damage and prolonged checkpoint-mediated arrest. It will be important to further address the regulation of different pools of $\mathrm{APC} / \mathrm{C}$ complexes and the role of Emi1 in future experiments.

Of note, the Cdc14B-Cdh1-Plk1 axis is activated in parallel to ATM, and failure of this pathway is not compensated by ATM-dependent checkpoint signaling. ${ }^{45}$ It is well documented that mutations in ATM have deleterious consequences in terms of acquisition of genomic instability and tumor development (as described in patients with Ataxia teleangiectasia and ATM $-/-$ mouse models). ${ }^{56}$ In particular, hematological malignancies are associated with ATM mutation. Therefore, deregulation of the Cdc14B-Cdh1-Plk1 pathway may be 
critically important in tumorigenesis as well. Remarkably, searches of the Oncomine database for differential expression of Cdc14B, Cdh1, and Plk1 in normal versus tumor tissues showed a significant decrease of Cdc14B and Cdh1 in many common tumors, such as breast, prostate, ovary, liver, and brain tumors. ${ }^{45}$ Conversely, levels of Plk1 are significantly increased in most tumors. ${ }^{26,45}$ Thus, failure of the Cdc14B-Cdh1-Plk1 axis may be a relevant oncogenic event in various tumors.

\section{Checkpoint recovery}

The primary function of DNA damage checkpoints is to generate a timeframe for subsequent DNA damage repair. If this task can be accomplished, the cell can resume cell-cycle progression. In the G2 checkpoint, recovery eventually results in mitotic entry. Although the machinery that senses DNA damage, activates checkpoints, and executes damage repair has been studied to a significant extent, less is known about how cells recover from the checkpoint and terminate damage signaling.

Several recent studies showed that ubiquitin-proteasomedependent degradation of Claspin and Wee1 is an integral part of the G2 checkpoint recovery process. The relevant ubiquitin ligase in this process is the $\mathrm{SCF}^{\beta \operatorname{TrCP}}$ complex, which targets both proteins for degradation. ${ }^{4-49}$ Although removal of Claspin results in the termination of Chk1 signaling (leading to stabilization of Cdc25A), Wee1 degradation is the direct removal of the major Cdk1 inhibitory kinase. $\beta$ TrCP thus functions as a critical intersection at the G2 checkpoint, transmitting either activation (degradation of Cdc25A to establish the checkpoint) or inhibition (degradation of Claspin and Wee1 to recover from the checkpoint) signals. The key to this differential function lies in the strict dependency on substrate phosphorylation within the degron. Although phosphorylation of the Cdc25A degron is Chk1-dependent (but Chk1 does not phosphorylate the degron directly), Plk1 directly phosphorylates Claspin and Wee1 within the canonical $\beta$ TrCP recognition motif, thus initiating the degradation process (Figure 3).

Recently, Plk1- $\beta$ TrCP-mediated degradation has been implicated in the regulation of the Fanconi Anemia (FA) pathway. The FA pathway, in which at least 13 different proteins participate, is an important pathway that ensures error-free DNA replication. Inactivating mutations in the corresponding genes underlie the inherited disease FA (reviewed in References ${ }^{7,57}$ ). Plk1-mediated phosphorylation of FANCM, an integral part of the FA complex, which loads the FA core onto chromatin, has now been shown to result in $\beta$ TrCP-mediated degradation of FANCM at G2/M. ${ }^{58}$ This mechanism facilitates the timely termination of FA-mediated DNA repair activity, contributing to faithful checkpoint recovery.

The various functions of Plk1 in pro-mitotic signaling give rise to the essential question of how Plk1 is reactivated during checkpoint recovery. In response to the DNA damage, Plk1 is kept in check by $\mathrm{APC} / \mathrm{C}^{\mathrm{Cdh} 1}$-dependent degradation, a process initiated by Cdc14B (see above). ${ }^{45}$ Additionally, the activating phosphorylation of Plk1 is also inhibited by a still unknown mechanism. $^{59}$ In a recent report, $\mathrm{APC} / \mathrm{C}^{\mathrm{Cdh} 1}$. mediated degradation of Cdc5, the budding yeast homolog of Plk1, was found to inactivate Cdc14 after mitotic exit by promoting its return to the nucleolus. ${ }^{60}$ It may be possible that a similar mechanism exists in mammalian cells, and it is extended to the function of Cdc14B in the G2 DNA damage response. In this manner, the Cdc14B-Cdh1-Plk1 axis could work as a feedback loop that regulates both activation and inactivation of the $\mathrm{G} 2$ checkpoint.

Finally, $\beta$ TrCP-mediated degradation may also be involved in the regulation of the critical cell fate decision after DNA damage: whether to resume the cell cycle or induce apoptosis. This possibility is based on the recent identification of the pro-apoptotic molecule, BimEL, as a substrate of $\beta$ TrCP. ${ }^{61}$ Moreover, $\beta$ TrCP has been implicated in the Mdm2-independent degradation of $\mathrm{p} 53$, which is initiated by IKK2-mediated phosphorylation of p53. ${ }^{62}$ Whether the G2 checkpoint makes use of these pathways will be subject of future investigations.

\section{Role of deubiquitylating enzymes (DUBs) in regulating the G2 checkpoint}

The reversible nature of ubiquitylation has brought deubiquitylation into the focus of cell cycle and checkpoint regulation. The human genome harbors approximately 90 deubiquitylating enzymes (DUBs), which cleave ubiquitin off substrates, thereby terminating ubiquitin-dependent processes. ${ }^{63-65}$ However, few DUBs have been assigned functions or substrates. Present evidence suggests that DUBs are highly regulated and function with a level of specificity, making them comparable to ubiquitin ligases; thus, underscoring a model in which ubiquitylation and deubiquitylation are controlled by the balance between two opposing enzymes.

Several DUBs have been implicated in the DNA damage response, including USP1, USP7 (also known as HAUSP), and USP28. ${ }^{66-69}$ With regard to the G2 DNA damage checkpoint, USP7 and USP28 are particularly relevant by virtue of their differential action on the checkpoint mediator protein Claspin. ${ }^{68,69}$ Claspin, which is a substrate of APC/ $\mathrm{C}^{\text {Cdh1 } 1}$ in $\mathrm{G} 1$, is protected by USP28 from APC/C ${ }^{\text {Cdh1 }}$ mediated ubiquitylation upon activation of the G2 checkpoint. ${ }^{45}$ Moreover, USP28 has been shown to be activated in response to DNA damage to stabilize additional proteins of the DNA damage checkpoint pathway (e.g. 53BP1 and Chk2) and promote DNA damage-induced apoptosis through regulation of the pro-apoptotic Chk2-p53-PUMA pathway. ${ }^{69}$ Finally, steady-state levels of Claspin in G2 are controlled by USP7, which specifically counteracts SCF ${ }^{\beta \operatorname{TrCP}_{-}}$-mediated ubiquitylation of Claspin. ${ }^{68}$ Thus, though USP28-mediated deubiquitylation of Claspin seems vital for the initiation and early maintenance processes of the checkpoint, USP7 controls checkpoint duration and the timing of recovery, particularly when Plk1 re-accumulates and becomes active.

The case of USP28 and USP7 as independent DUBs for Claspin indicates high levels of specificity for these deubiquitylation events and suggests that DUBs may be specific not only for their substrates but also for distinct ubiquitylation events within the substrate. Moreover, the counterplay of USP28 and Cdh1 (with USP28 protecting only some substrates of $\mathrm{APC} / \mathrm{C}^{\mathrm{Cdh} 1}$ ) suggests that deubiquitylation is an important regulatory mechanism that guarantees specificity of substrate degradation by single ubiquitin ligases. 


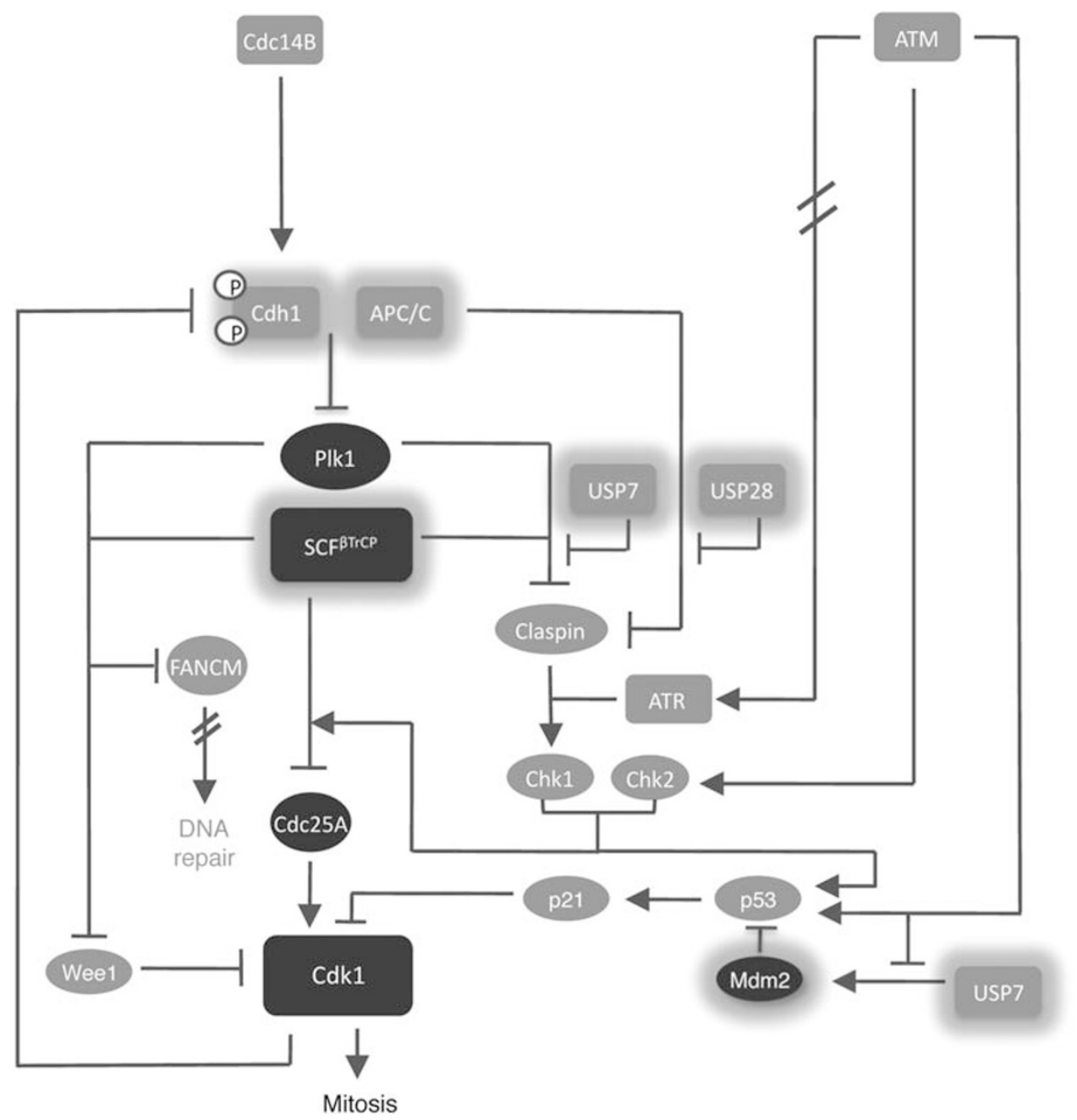

Figure 3 The role of ubiquitin ligases and deubiquitylating enzymes (DUBs) in the G2 checkpoint recovery process. After successful DNA repair in G2, cells recover from the checkpoint and proceed into mitosis. An integral part of this process is the degradation of Claspin and Wee1 through SCF ${ }^{\beta T C P}$, which is initiated by Plk1 after reconstitution of Plk1 expression and activity in the recovery process. The exact mechanism of Plk1 reactivation is presently unclear, but it may involve the inactivation of Cdc14B and $\mathrm{APC} / \mathrm{C}^{\mathrm{Cdh} 1}$, as well as activating phosphorylation of Plk1. Efficient Claspin degradation necessitates the removal of the stabilizing activities of USP7 and USP28 by still unknown mechanisms. Eventually, degradation of Claspin blocks Chk1 activation, allowing Cdc25A accumulation and activation of Cdk1. The activated PIk1-SCF ${ }^{\beta T r C P}$ hub additionally drives the degradation of FANCM, thus terminating DNA repair by the FA pathway in mitosis. Finally, inactivation of ATM results in the inactivation of ATR and Chk2, stabilization of Mdm2, and destabilization of p53, with subsequent loss of p21 transcription

Finally, two other functions of USP7 and USP28 in checkpoint control should be mentioned. First, USP7 functions as a DUB for Mdm2, the ubiquitin ligase for p53. ${ }^{65,70-72}$ In response to DNA damage, ATM phosphorylates Mdm2, thereby lowering its affinity for USP7, resulting in more rapid Mdm2 degradation. As a result, less p53 is ubiquitylated and degraded. Thus, USP7 participates in controlling the abundance of p53 during cell-cycle checkpoints. Second, USP28 seems to regulate the stability of substrates of the $\mathrm{SCF}^{\mathrm{Fbxw} 7}$ ligase, which functions as a tumor suppressor. This function may be relevant for the G2 checkpoint because DNA damage triggers dissociation of USP28 from c-Myc (a substrate of SCF ${ }^{\mathrm{Fbxw7}}$ ), thus triggering its degradation and stalling cell-cycle progression. ${ }^{73,74}$ In line with its function in counteracting degradation of c-Myc, USP28 has been shown to be overexpressed in colon and breast carcinomas. ${ }^{74}$ Thus, USP28 may possess the properties of both a tumor suppressor and an oncoprotein. Further experiments are necessary to elucidate this point.

\section{Concluding remarks and clinical outlook}

The recent progress reviewed herein provides evidence of the emerging role of the UPS, particularly the SCF and APC/C ligases, in regulating the G2 DNA damage checkpoint (Figures 2 and 3). The involvement of $A P C / C^{C d h 1}$ in the $\mathrm{G} 2$ DNA damage checkpoint gives rise to several interesting questions. To date, the most upstream signaling component of $\mathrm{APC} / \mathrm{C}^{\mathrm{Cdh} 1}$ reactivation is $\mathrm{Cdc} 14 \mathrm{~B}$, whose activation is operated in parallel to ATM. Thus, the initial event, which includes release from the nucleolus to allow nuclear translocation, deserves closer scrutiny. Another question concerns the substrates degraded through $\mathrm{APC} / \mathrm{C}^{\mathrm{Cdh} 1}$ in response to genotoxic stresses. Currently, the only identified substrate is 
Plk1, but other substrates may also be involved. The quest for other candidate substrates may identify unexpected proteins, which have not been associated earlier with Cdh1 (in late mitosis and G1). ${ }^{45}$ USP28 is at least partially involved in this process through deubiquitylation of Claspin and potentially other Cdh1 substrates. As research on the large family of DUBs continues, other DUBs may soon be added to this list.

The G2 checkpoint is also attractive from the point of view of targeted tumor therapy. DNA damage checkpoints work to repair DNA and to promote survival of the organism, but they might also reduce the effectiveness of various cancer treatments, which frequently induce DNA damage. ${ }^{75}$ If the activity of the checkpoint and DNA repair could be selectively reduced in tumor cells, the efficiency of treatment could be increased (chemosensitation). On the other hand, if the checkpoint response and repair could be increased selectively in normal, proliferating cells, while inducing apoptosis in tumor cells, therapies could be optimized to reduce side effects (chemoprotection and antitumor activity). ${ }^{75}$ Modulation of the G2 DNA damage checkpoint could potentially achieve both of these objectives. The role of the G2 checkpoint as a key guardian of the cancer cell genome has clearly emerged in the last 15 years, thus making G2 abrogation strategies attractive. Such an approach could prevent cancer cells from repairing DNA damage, forcing them into $M$ phase and subsequently into mitotic catastrophe and apoptosis. Overexpression of proteins that undermine the G2 checkpoint and drive cells into mitosis has been observed in different types of cancer (e.g. Plk1 and Cdc25A), presenting target candidates to directly induce antitumor activity. Indeed, inhibitors of Plk1 have shown robust antiproliferative activity in various tumors with overexpression of Plk1 (e.g. acute myelogenous leukemia), and clinical trials are currently being conducted to further evaluate this therapeutic principle. ${ }^{76,77}$

Compounds inhibiting proteasomal degradation have recently been proven effective in different types of tumors. Much of the current evidence for the efficacy of proateasome inhibition is derived from the successful clinical introduction of bortezomib, a compound that should inhibit all ubiquitin ligases by inhibiting the degradation of target proteins by the $26 \mathrm{~S}$ proteasome. ${ }^{78}$ Given the wide range of cellular processes regulated by the UPS, a targeted approach to specific ubiquitiylation events would be predicted to achieve higher therapeutic ratios. Moreover, the presence of more than 500 E3 ligases and approximately 100 DUBs in human cells makes it plausible that the alterations of the ubiquitin system identified in cancer so far offer merely a glimpse of a much more widespread mechanistic hub, whose deregulation significantly contributes to tumorigenesis. Ubiquitin ligases may represent effective targets because of their intrinsic specificity for particular substrates. At present, Mdm2 (a ubiquitin ligase that regulates the stability of p53) is one of the few ligases that have been shown to be amenable to specific inhibition. Although lead compounds have not yet been developed into clinically relevant therapeutics, the initial studies have shown that specific inhibition of ligases is indeed feasible. ${ }^{79,80}$ The major shortcoming of the ubquitin ligase inhibition strategy is that effective compounds must block the physical interaction between an E3 and its substrate, a difficult challenge that will have to be addressed. This approach will depend on a clear understanding of the structural basis of E3-substrate interactions, but such information is not yet readily available for most of the E3s. As ongoing research in the UPS field renders more and more answers to these open questions, the quest for compounds specifically targeting the UPS will hopefully yield novel classes of cancer drugs, which will provide powerful tools for targeted and specific tumor therapy.

Acknowledgements. We thank Christine von Klitzing and Jeff Skaar for critically reading the manuscript. We apologize to colleagues whose work could not be mentioned owing to space limitations. FB is supported by a fellowship from the AACR and the Emmy Noether Program of the German Research Foundation, and MP by grants from NIH and MMRF. MP is an investigator with the Howard Hughes Medical Institute.

1. Bakkenist CJ, Kastan MB. Initiating cellular stress responses. Cell 2004; 118: 9-17.

2. Harper JW, Elledge SJ. The DNA damage response: ten years after. Mol Cell 2007; 28: 739-745.

3. Kastan MB, Bartek J. Cell-cycle checkpoints and cancer. Nature 2004; 432: 316-323.

4. Branzei D, Foiani M. Regulation of DNA repair throughout the cell cycle. Nat Rev Mol Cell Biol 2008; 9: 297-308

5. Lukas J, Lukas C, Bartek J. Mammalian cell cycle checkpoints: signalling pathways and their organization in space and time. DNA Repair (Amst) 2004; 3: 997-1007.

6. Hakem R. DNA-damage repair; the good, the bad, and the ugly. EMBO $J 2008 ; 27$ : 589-605.

7. Kennedy RD, D'ndrea AD. DNA repair pathways in clinical practice: lessons from pediatric cancer susceptibility syndromes. J Clin Oncol 2006; 24: 3799-3808.

8. Lee JH, Paull TT. ATM activation by DNA double-strand breaks through the Mre11-Rad50Nbs1 complex. Science 2005; 308: 551-554.

9. Falck J, Coates J, Jackson SP. Conserved modes of recruitment of ATM, ATR and DNA-PKcs to sites of DNA damage. Nature 2005; 434: 605-611.

10. Jazayeri A, Falck J, Lukas C, Bartek J, Smith GC, Lukas J et al. ATM- and cell cycledependent regulation of ATR in response to DNA double-strand breaks. Nat Cell Biol 2006; 8: $37-45$.

11. Bartek J, Lukas J. DNA damage checkpoints: from initiation to recovery or adaptation. Curr Opin Cell Biol 2007; 19: 238-245.

12. Cardozo T, Pagano M. The SCF ubiquitin ligase: insights into a molecular machine. Nat Rev Mol Cell Biol 2004; 5: 739-751.

13. Peters JM. The anaphase promoting complex/cyclosome: a machine designed to destroy. Nat Rev Mol Cell Biol 2006; 7: 644-656.

14. Li M, Zhang P. The function of APC/CCdh1 in cell cycle and beyond. Cell Div 2009; 4: 2

15. Frescas $D$, Pagano M. Deregulated proteolysis by the F-box proteins SKP2 and beta-TrCP: tipping the scales of cancer. Nat Rev Cancer 2008; 8: 438-449.

16. Bassermann F, von Klitzing C, Illert AL, Munch S, Morris SW, Pagano M et al. Multisite phosphorylation of nuclear interaction partner of ALK (NIPA) at G2/M involves cyclin B1/Cdk1. J Biol Chem 2007; 282: 15965-15972.

17. Bassermann F, von Klitzing $\mathrm{C}$, Munch $\mathrm{S}$, Bai RY, Kawaguchi $\mathrm{H}$, Morris SW et al NIPA defines an SCF-type mammalian E3 ligase that regulates mitotic entry. Cell 2005; 122: 45-57.

18. Wei W, Ayad NG, Wan Y, Zhang GJ, Kirschner MW, Kaelin Jr WG. Degradation of the SCF component Skp2 in cell-cycle phase G1 by the anaphase-promoting complex. Nature 2004; 428: 194-198.

19. Bashir T, Dorrello NV, Amador V, Guardavaccaro D, Pagano M. Control of the SCF(Skp2Cks1) ubiquitin ligase by the APC/C(Cdh1) ubiquitin ligase. Nature 2004; 428: 190-193.

20. Benmaamar R, Pagano M. Involvement of the SCF complex in the control of Cdh1 degradation in S-phase. Cell Cycle 2005; 4: 1230-1232.

21. Guardavaccaro D, Pagano M. Stabilizers and destabilizers controlling cell cycle oscillators. Mol Cell 2006; 22: 1-4.

22. Kumagai A, Dunphy WG. Claspin, a novel protein required for the activation of Chk1 during a DNA replication checkpoint response in Xenopus egg extracts. Mol Cell 2000; 6 : 839-849.

23. Takaki T, Trenz K, Costanzo V, Petronczki M. Polo-like kinase 1 reaches beyond mitosis-cytokinesis, DNA damage response, and development. Curr Opin Cell Biol 2008; 20: $650-660$.

24. Busino L, Donzelli M, Chiesa M, Guardavaccaro D, Ganoth D, Dorrello NV et al. Degradation of Cdc25A by beta-TrCP during $S$ phase and in response to DNA damage. Nature 2003 2003/11/06/ 426: 87-91.

25. Jin J, Shirogane T, Xu L, Nalepa G, Qin J, Elledge SJ et al. SCFbeta-TRCP links Chk1 signaling to degradation of the Cdc25A protein phosphatase. Genes Dev 2003; 17 : 3062-3074. 
26. Sadhu K, Reed SI, Richardson H, Russell P. Human homolog of fission yeast cdc 25 mitotic inducer is predominantly expressed in G2. Proc Natl Acad Sci USA 1990; 87: 5139-5143.

27. Galaktionov K, Beach D. Specific activation of cdc25 tyrosine phosphatases by B-type cyclins: evidence for multiple roles of mitotic cyclins. Cell 1991; 67: 1181-1194.

28. Boutros R, Lobjois V, Ducommun B. CDC25 phosphatases in cancer cells: key players? Good targets? Nat Rev Cancer 2007; 7: 495-507.

29. Nagata A, Igarashi M, Jinno S, Suto K, Okayama $H$. An additional homolog of the fission yeast cdc25+ gene occurs in humans and is highly expressed in some cancer cells. New Biol 1991; 3: 959-968.

30. Lammer C, Wagerer S, Saffrich R, Mertens D, Ansorge W, Hoffmann I. The cdc25B phosphatase is essential for the G2/M phase transition in human cells. J Cell Sci 1998; 111 (Pt 16): 2445-2453.

31. Perdiguero E, Nebreda AR. Regulation of Cdc25C activity during the meiotic G2/M transition. Cell Cycle 2004; 3: 733-737.

32. Mailand N, Podtelejnikov AV, Groth A, Mann M, Bartek J, Lukas J. Regulation of G(2)/M events by $\mathrm{Cdc} 25 \mathrm{~A}$ through phosphorylation-dependent modulation of its stability. EMBO 2002; 21: 5911-5920.

33. Hoffmann I, Draetta G, Karsenti E. Activation of the phosphatase activity of human cdc25A by a cdk2-cyclin $E$ dependent phosphorylation at the G1/S transition. EMBO J 1994; 13 $4302-4310$.

34. Ray D, Terao Y, Nimbalkar D, Hirai H, Osmundson EC, Zou X et al. Hemizygous disruption of Cdc25A inhibits cellular transformation and mammary tumorigenesis in mice. Cancer Res 2007; 67: 6605-6611.

35. Ferguson AM, White LS, Donovan PJ, Piwnica-Worms H. Normal cell cycle and checkpoint responses in mice and cells lacking $\mathrm{Cdc} 25 \mathrm{~B}$ and $\mathrm{Cdc} 25 \mathrm{C}$ protein phosphatases. $\mathrm{Mol} \mathrm{Cell}$ Biol 2005; 25: 2853-2860.

36. Busino L, Chiesa M, Draetta GF, Donzelli M. Cdc25A phosphatase: combinatorial phosphorylation, ubiquitylation and proteolysis. Oncogene 2004; 23: 2050-2056.

37. Ray D, Terao Y, Fuhrken PG, Ma ZQ, DeMayo FJ, Christov K et al. Deregulated CDC25A expression promotes mammary tumorigenesis with genomic instability. Cancer Res 2007; 67: 984-991.

38. Lukas C, Sorensen CS, Kramer E, Santoni-Rugiu E, Lindeneg C, Peters JM et al Accumulation of cyclin $\mathrm{B} 1$ requires $\mathrm{E} 2 \mathrm{~F}$ and cyclin-A-dependent rearrangement of the anaphase-promoting complex. Nature 1999; 401: 815-818.

39. Sorensen CS, Lukas C, Kramer ER, Peters JM, Bartek J, Lukas J. A conserved cyclinbinding domain determines functional interplay between anaphase-promoting complexCdh1 and cyclin A-Cdk2 during cell cycle progression. Mol Cell Biol 2001; 21: 3692-3703.

40. Mitra J, Enders GH, Azizkhan-Clifford J, Lengel KL. Dual regulation of the anaphase promoting complex in human cells by cyclin A-Cdk2 and cyclin A-Cdk1 complexes. Cell Cycle 2006; 5: 661-666.

41. Di Fiore B, Pines J. Defining the role of Emi1 in the DNA replication-segregation cycle. Chromosoma 2008; 117: 333-338.

42. Miller JJ, Summers MK, Hansen DV, Nachury MV, Lehman NL, Loktev A et al. Emi1 stably binds and inhibits the anaphase-promoting complex/cyclosome as a pseudosubstrate inhibitor. Genes Dev 2006; 20: 2410-2420.

43. Rape M, Kirschner MW. Autonomous regulation of the anaphase-promoting complex couples mitosis to S-phase entry. Nature 2004; 432: 588-595.

44. Sudo T, Ota Y, Kotani S, Nakao M, Takami Y, Takeda S et al. Activation of Cdh1dependent APC is required for G1 cell cycle arrest and DNA damage-induced G2 checkpoint in vertebrate cells. EMBO J 2001; 20: 6499-6508.

45. Bassermann F, Frescas D, Guardavaccaro D, Busino L, Peschiaroli A, Pagano M. The Cdc14B-Cdh1-Plk1 axis controls the G2 DNA-damage-response checkpoint. Cell 2008 134: 256-267.

46. Watanabe N, Arai H, Nishihara Y, Taniguchi M, Watanabe N, Hunter T et al. M-phase kinases induce phospho-dependent ubiquitination of somatic Wee1 by SCFbeta-TrCP. Proc Natl Acad Sci USA 2004; 101: 4419-4424.

47. Mamely I, van Vugt MA, Smits VA, Semple JI, Lemmens B, Perrakis A et al. Polo-like kinase-1 controls proteasome-dependent degradation of Claspin during checkpoint recovery. Curr Biol 2006; 16: 1950-1955.

48. Mailand N, Bekker-Jensen S, Bartek J, Lukas J. Destruction of Claspin by SCFbetaTrCP restrains Chk1 activation and facilitates recovery from genotoxic stress. Mol Cell 2006; 23: 307-318.

49. Peschiaroli A, Dorrello NV, Guardavaccaro D, Venere M, Halazonetis T, Sherman NE et al. SCFbetaTrCP-mediated degradation of Claspin regulates recovery from the DNA replication checkpoint response. Mol Cell 2006; 23: 319-329.

50. Garcia-Higuera I, Manchado E, Dubus P, Canamero M, Mendez J, Moreno S et al. Genomic stability and tumour suppression by the APC/C cofactor Cdh1. Nat Cell Biol 2008; 10: $802-811$
51. Skaar JR, Pagano M. Cdh1: a master G0/G1 regulator. Nat Cell Biol 2008; 10: 755-757.

52. Stegmeier F, Amon A. Closing mitosis: the functions of the Cdc14 phosphatase and its regulation. Annu Rev Genet 2004; 38: 203-232.

53. D'mours D, Amon A. At the interface between signaling and executing anaphase-Cdc14 and the FEAR network. Genes Dev 2004; 18: 2581-2595.

54. Sullivan M, Morgan DO. Finishing mitosis, one step at a time. Nat Rev Mol Cell Biol 2007; 8: 894-903.

55. Lee J, Kim JA, Barbier V, Fotedar A, Fotedar R. DNA damage triggers p21WAF1dependent Emi1 down-regulation that maintains G2 arrest. Mol Biol Cell 2009; 20: 1891-1902.

56. Lavin MF. Ataxia-telangiectasia: from a rare disorder to a paradigm for cell signalling and cancer. Nat Rev Mol Cell Biol 2008; 9: 759-769.

57. de Winter JP, Joenje H. The genetic and molecular basis of Fanconi anemia. Mutat Res 2009; 668: 11-19.

58. Kee Y, Kim JM, D'ndrea A. Regulated degradation of FANCM in the Fanconi anemia pathway during mitosis. Genes Dev 2009; 23: 555-560.

59. Smits VA, Klompmaker R, Arnaud L, Rijksen G, Nigg EA, Medema RH. Polo-like kinase-1 is a target of the DNA damage checkpoint. Nat Cell Biol 2000; 2: 672-676.

60. Visintin C, Tomson BN, Rahal R, Paulson J, Cohen M, Taunton J et al. APC/C-Cdh1mediated degradation of the Polo kinase $\mathrm{Cdc5}$ promotes the return of $\mathrm{Cdc} 14$ into the nucleolus. Genes Dev 2008; 22: 79-90.

61. Dehan E, Bassermann F, Guardavaccaro D, Vasiliver-Shamis G, Cohen M, Lowes KN et al. betaTrCP- and Rsk1/2-mediated degradation of BimEL inhibits apoptosis. Mol Cell 2009; 33: 109-116.

62. Xia Y, Padre RC, De Mendoza TH, Bottero V, Tergaonkar VB, Verma IM. Phosphorylation of p53 by lkappaB kinase 2 promotes its degradation by beta-TrCP. Proc Natl Acad Sci USA 2009; 106: 2629-2634

63. Amerik AY, Hochstrasser M. Mechanism and function of deubiquitinating enzymes. Biochim Biophys Acta 2004; 1695: 189-207.

64. Nijman SM, Luna-Vargas MP, Velds A, Brummelkamp TR, Dirac AM, Sixma TK et al. A genomic and functional inventory of deubiquitinating enzymes. Cell 2005; 123: 773-786.

65. Song L, Rape M. Reverse the curse-the role of deubiquitination in cell cycle control. Curr Opin Cell Biol 2008; 20: 156-163.

66. Nijman SM, Huang TT, Dirac AM, Brummelkamp TR, Kerkhoven RM, D'Andrea AD et al. The deubiquitinating enzyme USP1 regulates the Fanconi anemia pathway. Mol Cell 2005; 17: $331-339$.

67. Huang TT, Nijman SM, Mirchandani KD, Galardy PJ, Cohn MA, Haas W et al. Regulation of monoubiquitinated PCNA by DUB autocleavage. Nat Cell Biol 2006; 8: 339-347.

68. Faustrup H, Bekker-Jensen S, Bartek J, Lukas J, Mailand N. USP7 counteracts SCFbetaTrCP- but not APCCdh1-mediated proteolysis of Claspin. J Cell Biol 2009; 184: 13-19.

69. Zhang D, Zaugg K, Mak TW, Elledge SJ. A role for the deubiquitinating enzyme USP28 in control of the DNA-damage response. Cell 2006; 126: 529-542.

70. Li M, Brooks CL, Kon N, Gu W. A dynamic role of HAUSP in the p53-Mdm2 pathway. Mol Cell 2004: 13: 879-886.

71. Meulmeester E, Maurice MM, Boutell C, Teunisse AF, Ovaa H, Abraham TE et al. Loss of HAUSP-mediated deubiquitination contributes to DNA damage-induced destabilization of Hdmx and Hdm2. Mol Cell 2005; 18: 565-576.

72. Lahav G, Rosenfeld N, Sigal A, Geva-Zatorsky N, Levine AJ, Elowitz MB et al. Dynamics of the p53-Mdm2 feedback loop in individual cells. Nat Genet 2004; 36: 147-150.

73. Popov N, Herold S, Llamazares M, Schulein C, Eilers M. Fbw7 and Usp28 regulate myc protein stability in response to DNA damage. Cell Cycle 2007; 6: 2327-2331.

74. Popov N, Wanzel M, Madiredjo M, Zhang D, Beijersbergen R, Bernards R et al. The ubiquitin-specific protease USP28 is required for MYC stability. Nat Cell Biol 2007; 9: 765-774.

75. Zhou BB, Bartek J. Targeting the checkpoint kinases: chemosensitization versus chemoprotection. Nat Rev Cancer 2004; 4: 216-225.

76. Strebhardt K, Ullrich A. Targeting polo-like kinase 1 for cancer therapy. Nat Rev Cancer 2006; 6: 321-330.

77. Renner AG, Dos Santos C, Recher C, Bailly C, Creancier L, Kruczynski A et al. Polo-like kinase 1 is over-expressed in acute myeloid leukemia and its inhibition preferentially targets the proliferation of leukemic cells. Blood 2009; 114: 659-662.

78. Sterz J, von Metzler I, Hahne JC, Lamottke B, Rademacher J, Heider U et al. The potential of proteasome inhibitors in cancer therapy. Expert Opin Investig Drugs 2008; 17: 879-895.

79. Yang Y, Kitagaki J, Wang H, Hou DX, Perantoni AO. Targeting the ubiquitin-proteasome system for cancer therapy. Cancer Sci 2009; 100: 24-28.

80. Cardozo T, Pagano M. Wrenches in the works: drug discovery targeting the SCF ubiquitin ligase and APC/C complexes. BMC Biochem 2007; 8 (Suppl 1): S9. 